\title{
Digital micromirror device for holographic and Fourier optics applications
}

\author{
Brice Douet $^{1}$, Téo Tedoldi ${ }^{1}$, Adeline Kabacinski ${ }^{2}$, Ambra Morana ${ }^{3}$, Guilhem Gallot ${ }^{1, *}$, \\ and Raphaël Bouganne ${ }^{1}$ \\ ${ }^{1}$ Ecole polytechnique, Institut Polytechnique de Paris, 91128 Palaiseau, France \\ ${ }^{2}$ LOA, ENSTA-Paristech, CNRS, Ecole Polytechnique, Institut Polytechnique de Paris, 91762 Palaiseau, France \\ ${ }^{3}$ LULI, CNRS, CEA, Ecole Polytechnique, UPMC, Sorbonne Université, Institut Polytechnique de Paris, 91128 \\ Palaiseau, France
}

Received: 9 February 2021 / Accepted: 9 June 2021

\begin{abstract}
The electromagnetic wavefront diffracted by an object carries information about the shape of the object from which the wave was emitted. Being able to record the phase and intensity of such a wave thus allows to reconstruct the object from the information carried by the wave, even if the object is no longer present. Among the reconstruction techniques, holography plays a big part. However the waves may experience a great variety of distortions on their way from the object to the measurement apparatus. Thus being able to shape the wavefront at will is key in holography. Micromirror light modulators are powerful tools for that matter and are well known for holographic applications. This paper explores the fundamental principles for digitally reconstructing a precise image of an object, but also for digitally correcting an imperfectly shaped wavefront, by exploiting the diffraction properties of light on a reflective surface. The methods presented here have been implemented as part of practical work for 2nd year students at the Ecole Polytechnique (last year of undergraduate program).
\end{abstract}

Keywords: Holography / Digital Micromirror Device / Spatial light modulation.

\section{Introduction}

Holography is a process used to record both phase and amplitude of a light wave diffracted by an object. Having this information enables to reconstitute the stereoscopic relief of the object and to create a 3D image of it. This process was discovered 1948 by the Hungarian physicist Dennis Gabor [1], who was awarded the Nobel Prize in physics in 1971 for his invention. In order to record information about the phase of an electromagnetic wave, the oscillations of which are way to fast for available intensity detectors and which cannot be recorded directly, the interference of two coherent beams is generally used, the spatial amplitude modulation of which is directly related to the phase difference between the two incident beams. Generally, a coherent beam is divided into two arms, then recombined on a recording device (holographic plate or CCD sensor), to measure the interference of the reference beam and the beam diffracted by the object. To obtain this interference pattern, it is necessary that the optical path difference between the two arms of the interferometer is shorter than the coherence length of the source.

\footnotetext{
*e-mail: guilhem.gallot@polytechnique.edu
}

The rise of digital holography goes along with the popularization of the use of CCD devices, which thus become widely available for industrial applications. Unlike analog holography, this method presents the advantages of reusable equipment (no recording plates, no chemicals), a less complex recording process (shorter exposure time, hence a lower sensibility to disturbances and vibrations) and a reading of the results independent from the experimental setup [2].

Another application of holography consists in the modification at will of a light wave both in amplitude and phase. In this paper we use an array of rotating micromirrors, called Digital Micromirror Device (DMD), which acts as a blazed grating and allows a spatial modulation of light by arbitrarily shaping the incident wavefront $[3-5]$. The shaped light field can then be recorded either by direct imaging of the DMD, or by Fourier plane imaging. DMDs are becoming increasingly used in experimental physics, such as in the field of cold atoms [6], topological physics [7], or imaging in complex and scattering environments [8].

These different methods have been implemented as part of practical work for second year students at the Ecole Polytechnique (last year of undergraduate program). In 


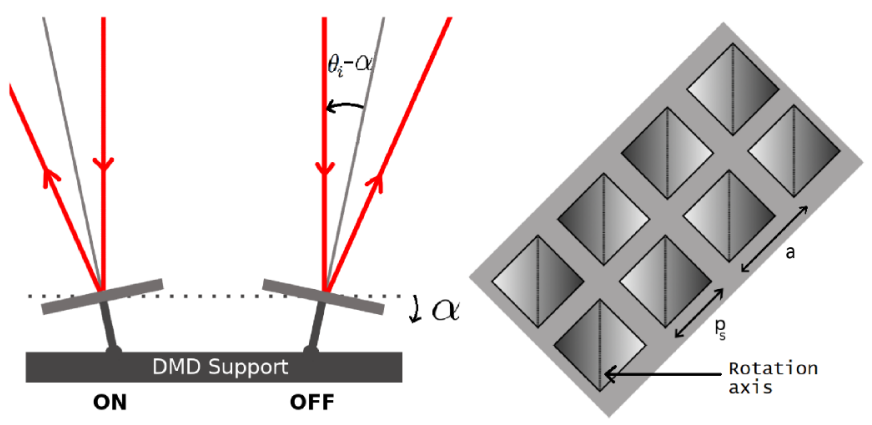

Fig. 1. Operating principle of the DMD. On the left, the two $\mathrm{ON}$ and $\mathrm{OFF}$ modes of a micromirror. Each micromirror rotates on its diagonal therefore the DMD is rotated at 450 in order to keep the incident beam parallel to the table.

this context, digital holography allows students to become familiar with the fundamental concepts of Fourier optics, optical coherence and lasers.

\section{Digital Micromirror Device (DMD)}

The DMD is an array of square micromirrors (pitch $a=$ $13.68 \mu \mathrm{m}$ ), each of which can rotate around one of its diagonal axes, with an angle $\alpha= \pm 12^{\circ}$ with respect to the plane of the DMD . These two configurations define the ON and OFF modes, as shown in Figure 1. The choice of the mode of each of the micromirrors allows a controlled modification of the wavefront of the reflected beam. The spatial selection of the light is done by collecting the light in the direction of reflection of one of these modes. In order to keep the incident beam parallel to the table, the DMD is rotated $45^{\circ}$ around its normal.

In a simple approach, the DMD in ON mode can be modelled as the convolution of a square micromirror $R$ of size $p_{s}$ with a grid $G r(x, y)$ defining the position of each mirror. Therefore, $D M D(x, y)=(R * G r)(x, y)$ with $R(x, y)=1, \forall(x, y) \in\left[-p_{s} / 2, p_{s} / 2\right]$ and 0 elsewhere, and $G r(x, y)=\sum_{i, j} \delta(x-i a, y-j a)$, where $\delta$ is Dirac's function and $a$ the period of the grating. The far-field distribution of the reflected light can thus be expressed as the Fourier transform of this convolution product, i.e. the product of a sinc function and the function $\mathcal{F}[G r]$. The diffracted orders are therefore Dirac peaks whose positions are defined as follows along the principal directions of the grating:

$$
\sin \theta_{i}+\sin \theta_{m}=\frac{m \lambda}{a}
$$

with $a$ the grating period, $\theta_{i}$ the angle of incidence, $\theta_{m}$ the angle of reflection with respect to the DMD normal for the diffracted order $m$. In reality, the DMD matrix is not an infinite grid. As a consequence, the diffraction pattern is not an ideal grid of Dirac functions but a grid of sinc. Experimentally, the DMD is rotated at $45^{\circ}$ since the axis of rotation of each micro-mirror is along its diagonal. The incident beam is set at $\theta_{i}=-31.3^{\circ}$ from the DMD to meet the blazing condition which allows to return the maximum intensity at a given order (in our case the order is $m=6$ ). The blazing angle is the angle of incidence for which the angle of a given diffracted order corresponds to the angle of specular reflection $\theta_{r}=2 \alpha-\theta_{i}$.

Since the axis of incidence of the light being at $45^{\circ}$ from the grating axis, this DMD can be compared to a blazed grating, whose particularity is to send the maximum intensity in a non trivial order by correctly selecting the angle of incidence.

Thereafter, we use a Texas Instruments DLP7000 DMD to conduct our experiments. Depending on the desired wavefront at the output of the DMD, we used different imaging techniques which are discussed in the following sections.

\section{Direct imaging}

In the first geometry, the DMD can be imaged directly on a CCD sensor with an afocal system. Initially, the laser beam is cleaned by a first filter consisting of a microscope objective and a hole of about 10 microns, which generates a spherical wave. This wave is then reflected on the DMD, then passes through an afocal system where the image and object focal points of the two lenses coincide. At this point, a second spatial filter is applied, which acts as a low-pass filter and smooths the image. Knowing that the field at the focal point is the Fourier transform of the field at the DMD level, the use of an aperture allows to attenuate the high frequencies, the lowest frequencies being located in the center of the image.

This kind of setup enables to image large figures, with slowly varying or uniform intensity over large areas. When it comes to very sharp patterns, it is preferable to use a Fourier plane imaging setup.

\section{Fourier plane imaging}

Knowing that the object and the image plane of a lens are related by a Fourier transform relationship, the imaging of the DMD in reciprocal space can be done with the setup shown in Figure 2, which will be used later on.

\subsection{Demonstration of the modification of amplitude and phase of an incident wave by the DMD}

Used in the Fourier plane configuration, the DMD can modify both the amplitude and the phase of an incident wave. Indeed, considering a $1 \mathrm{D}$ grating of period $G$, slit width $W$ and centre $X_{c}$, the amplitude $A_{p}$ of the diffracted field in the order $p$ is proportional to the Fourier transform of one period of the grating by an ideal lens modelled by the function $\Gamma(x)$, which is equal to 1 between $x_{c}-w / 2$ and $x_{c}+w / 2$ and 0 elsewhere, where $w=W / G$ and $x_{c}=$ $X_{c} / G$ are the normalized width and centre (i.e. $w \in[0,1[$ and $x_{c} \in\left[-1 / 2,1 / 2\left[\right.\right.$ ). Hence $A_{p} \propto \int_{-\infty}^{\infty} \Gamma_{y}(x) e^{-i 2 \pi p x} d x$, and then

$$
A_{p} \propto e^{-i 2 \pi p x_{c}} \frac{\sin (\pi p w)}{\pi p}
$$




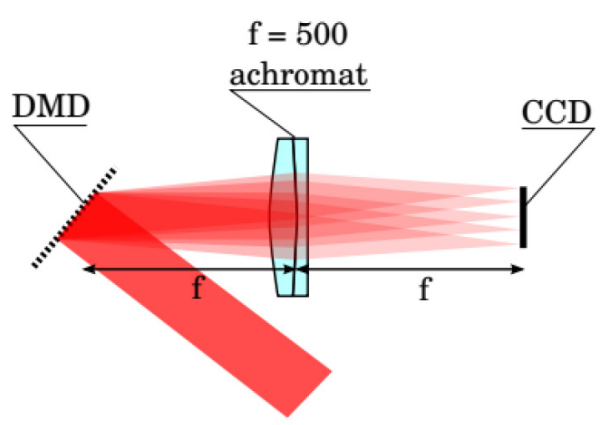

Fig. 2. Setup of the Fourier plane imaging of the DMD device. The CCD sensor is placed in the Fourier Plane of the lens, i.e. on the focal plane of the lens, symmetrically with respect to the DMD.
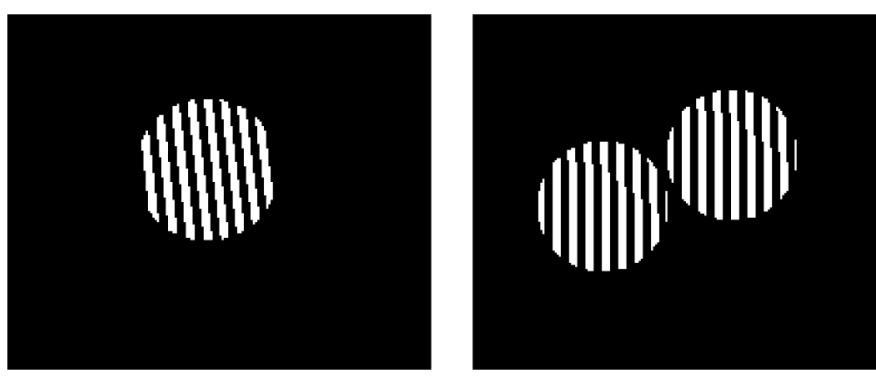

Fig. 3. On the left, small round grating displayed on the DMD to modulate the amplitude of diffracted orders, by changing its rotation, period and width. The round diameter is 32 pixels $(438 \mu \mathrm{m})$. On the right, two round gratings displayed on the DMD to modulate diffracted wave by changing their relative phase.

We notice that the grating is fully characterized by a width $w$ and a phase $\phi=2 \pi x_{c}$. This effect was visualized by displaying a small round grating on the DMD, as shown on the left of Figure 3 and by changing its properties, such as rotation, period and width. The results are given by Figure 4 .

One can observe, in reciprocal space, several diffracted orders resembling Airy disks. As expected, the width of the slits affects the brightness of the orders (Fig. 4ab), the maximum brightness being at $w=\frac{1}{2}$, which is consistent with equation (2) at order 1 . The tilt angle of the grating rotates the figure by the same angle (Fig. $4 \mathrm{c}$ and d). At last, increasing the spacing between the slits contracts horizontally the figure (Fig. 4e and f), since the position $X_{p}$ of order $p$ verifies $X_{p}=\frac{2 \pi f p}{k a}, f$ being the focal length of the lens and $k$ the wave vector.

In order to observe the effect of the phase, two gratings corresponding to the convolution of Young holes by a diffraction grating, shown on the right of Figure 3, are displayed on the DMD. The results are given by Figure 5 . On the top of the figure, one can observe the orders $-1,0$ and +1 diffracted by the DMD for two different dephasing between the two gratings ( $\Delta \phi=\frac{\pi}{2}$ to the left and $\Delta \phi=\pi$ to the right). The plots below represent the intensity profile of the first order, perpendicularly to the fringes for the corresponding dephasing. The dephasing between those
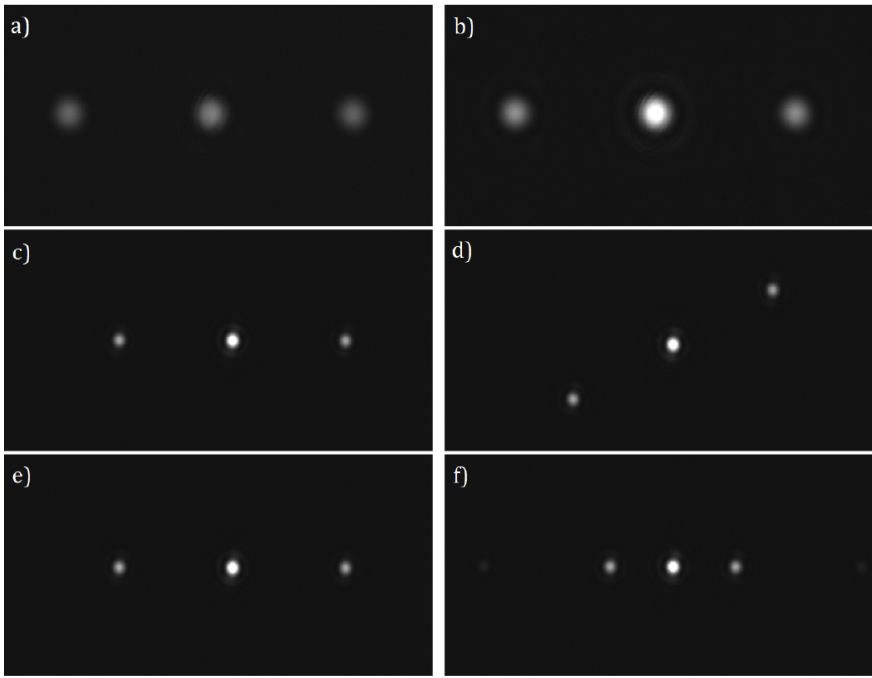

Fig. 4. Orders $-1,0$ and +1 diffracted by the DMD for different parameters of the displayed gratings. (a) and (b) The width of the slits varies from $W=26.3$ to $65.75 \mu \mathrm{m}$. (c) and (d) The angle of the grating varies from 0 to $\pi / 5$. (e) and (f) The period of the grating varies from $G=78.9$ to $131.5 \mu \mathrm{m}$.

two gratings strongly affects the position of interference fringes since the phase difference verifies

$$
\Delta \phi=\frac{2 \pi p \Delta x_{c}}{a},
$$

which is exactly what was shown experimentally. These results are thus consistent with equations 2 and 3 .

\subsection{Correction of the DMD wave front}

We saw in the previous section that the DMD is able to modify both the amplitude and the phase of an incident wave (for example by taking the first order diffracted mode of a displayed grating). We will demonstrate that, using the Fourier plane imaging setup, we are able to reconstruct various images in the plane of the CCD, by displaying on the DMD the computed inverse Fourier transform of a given image. In order to obtain an accurate image, the diffracted wavefront must only carry the information of what is displayed on the DMD chip. However, DMD are not ideal micromirror arrays. Imperfections like non-planarity of the DMD surface (which leads to phase disturbances), as well as inhomogeneous reflection coefficients of the micromirrors (which causes variations in intensity), disturb the diffracted light field.

We first looked at the diffraction pattern on the DMD without any correction by applying Figure 8a which represents a simple grating in a square smaller than the DMD itself. In the Fourier plane, we can therefore expect a bi-dimensional sinc distribution. What is observed in reality, due to the imperfections of the DMD, is shown in Figure 8b, and is not at all the expected distribution rather than a blurry big spot. Thus, a quantification of imperfections becomes mandatory.

In order to correct for the disturbances and obtain an accurate recorded image, we measured the defects in terms 

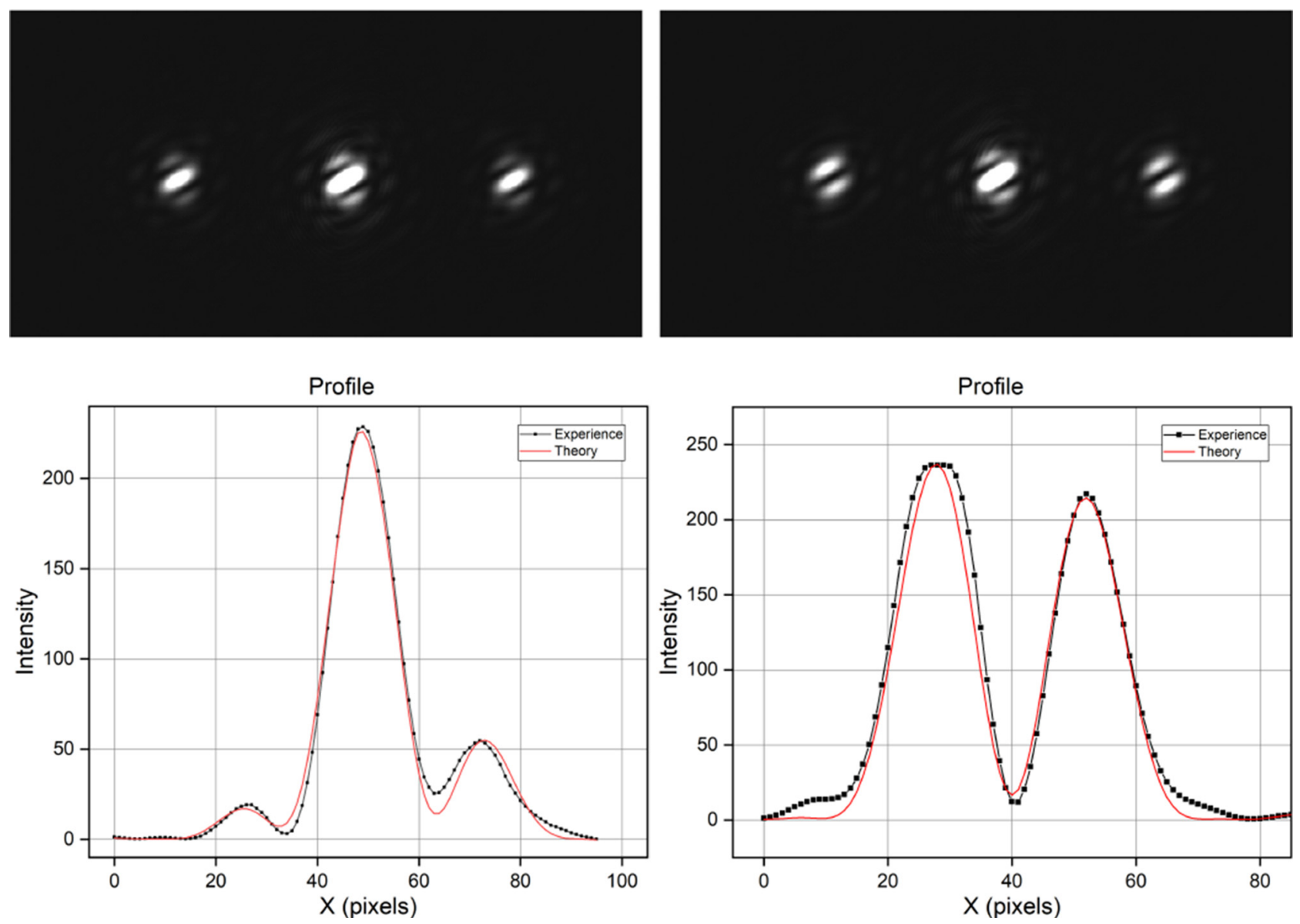

Fig. 5. On the top, orders $-1,0$ and +1 diffracted by the DMD with two gratings displayed. On the left, the dephasing is $\Delta \phi=\pi / 2$, on the right $\Delta \phi=\pi$. The plots below correspond to the intensity profile of the first order, perpendicularly to the fringes, for the two corresponding dephasings. Here $G=131.5 \mu \mathrm{m}$.

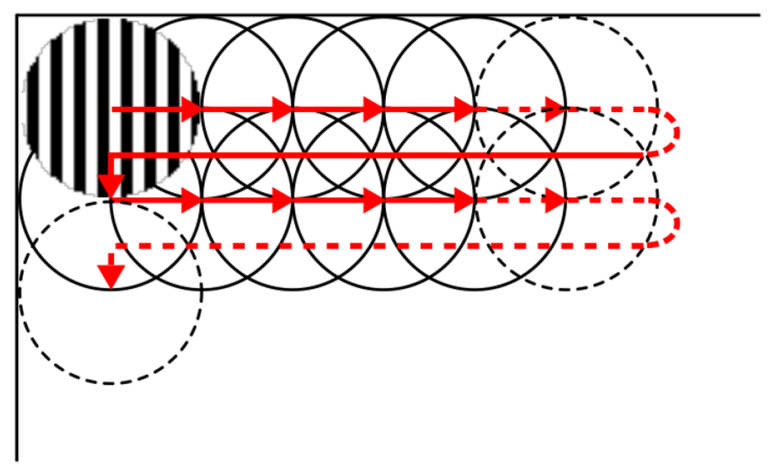

Fig. 6. Calibration of the DMD wavefront. Each pattern is composed of a round grating.

of intensity and phase over the entire surface of the DMD. This was done by sampling the entire surface of the DMD with a series of small circular gratings (geometry shown in Figure 6 [3]). A single displayed grating theoretically gives rise to an Airy disk on the CCD, the intensity and position of which provides information about the local reflection coefficient and surface deformation of the DMD. All grating patterns together cover the whole surface and also warrant overlapping redundancy. We recorded the diffraction pattern resulting from the display of each grating. By scanning the position of the grating on the DMD surface, the intensity and the position of Airy discs on the CCD are slightly altered, which reveals the variations in intensity and phase restitution on the surface of the DMD. Thus, by numerically recording the position of the first order and its intensity, information on local phase and intensity imperfections on the surface of the DMD can be deduced. The intensity profile of the wave is simply given by the measured amplitude of the Airy disks.

The phase we want to determine is the phase of the wave diffracted by the DMD, which corresponds to the incident phase on the DMD distorted by the aberrations introduced by the reflection on the DMD. This phase is measured experimentally from the local phase gradient. For each pattern applied on the DMD, we measure the distance between a reference position and the real position of the Airy disk produced by this pattern. Therefore, $(\delta \phi / \delta x, \delta \phi / \delta y)=k f(\Delta x, \Delta y)$. This principle is similar to the one used in Shack-Hartmann wavefront sensor [9]. The wavefront profile is therefore reconstructed by integrating the local gradient. A reference phase is chosen to be zero on a round pattern and then integrated step by step from 
(a) Interpolated intensity map

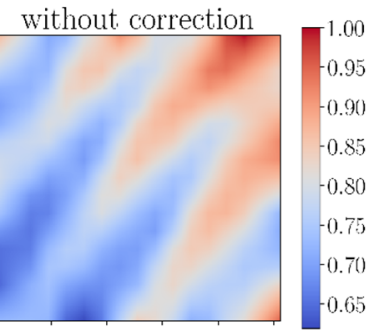

(c) Interpolated phase map

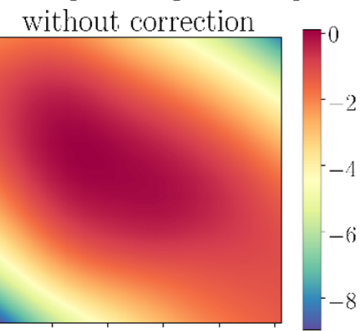

(b) Interpolated intensity map

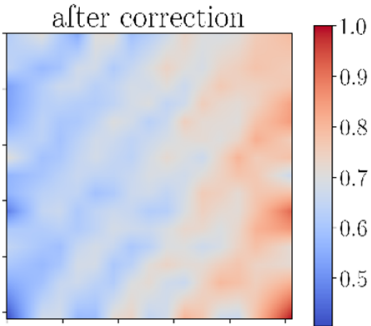

(d) Interpolated phase map

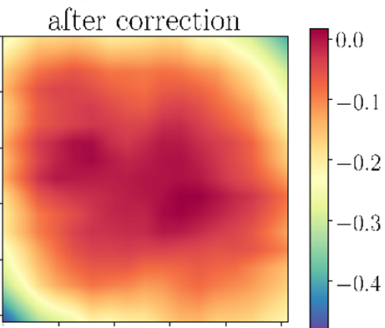

Fig. 7. Top: intensity maps, before and after correction, scaled by the DMD normalized intensity. Bottom: phase maps before and correction, scaled by the path difference divided by the laser wavelength. The width of the images is 800 pixels $(10.9 \mathrm{~mm})$.

it using the following Taylor expansion

$$
\phi(x+\Lambda, y+\Lambda)=\phi(x, y)+\frac{k \Lambda}{f}(\Delta x+\Delta y)
$$

where $\Lambda$ is the step between adjacent patterns. A linear interpolation is then used to adjust the phase profile on each pixel of the DMD. We can thus reconstitute the intensity and phase distortions on the mirror surface and establish calibrated intensity and phase maps of the DMD.

The intensity and phase maps (Figs. 7a and 7c) show huge distortions. The non-planarity of the DMD is highlighted by the phase maps where the DMD is shown to induce a path difference of $8 \lambda$ from the center to the edge of its surface, making it usable only near the center.

The previous method allowed us to establish phase and intensity maps of the DMD. They were fed back to the hologram displayed on the DMD in order to correct for the distortion (Fig. 8c). Then a new intensity and phase characterization was performed (Fig. $7 \mathrm{~b}$ and $7 \mathrm{~d}$ ). The established correction reduces the induced path difference to $\lambda / 3$, which represents an improvement by a factor of 20 . Intensity variations are reduced as well.

The output of this corrected grating provides a proper sinc distribution (see Fig. 8d). We then iterated the calibration process using this new grating. After 2 iterations, the process is precise enough to render correct images. The smoothed calibration maps have been taken into account to compute the inverse Fourier transform, considering the corrected wavefront information, as shown in Figures 8c and $8 \mathrm{e}$.

Wavefront correction for a simple grating was successfully used to reconstitute its Fourier transform. After respectively 1 and 2 iterations of the process described above, we obtained the results presented in Figure 8d and

a)

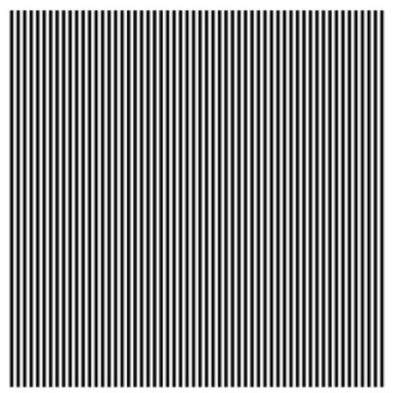

b)

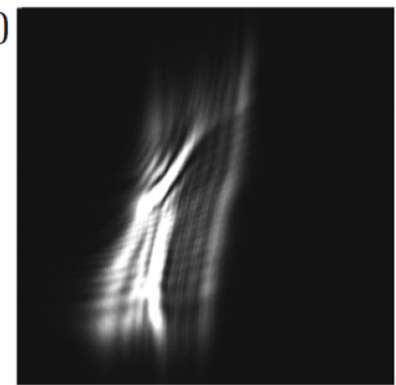

c)

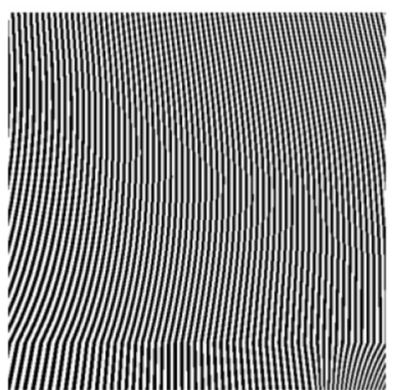

d)

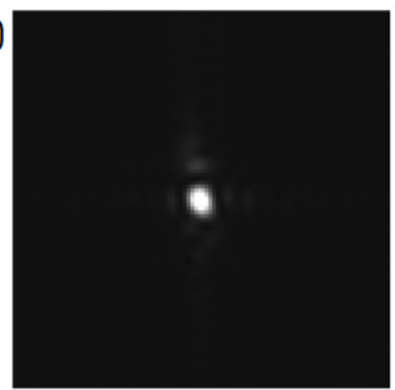

e)
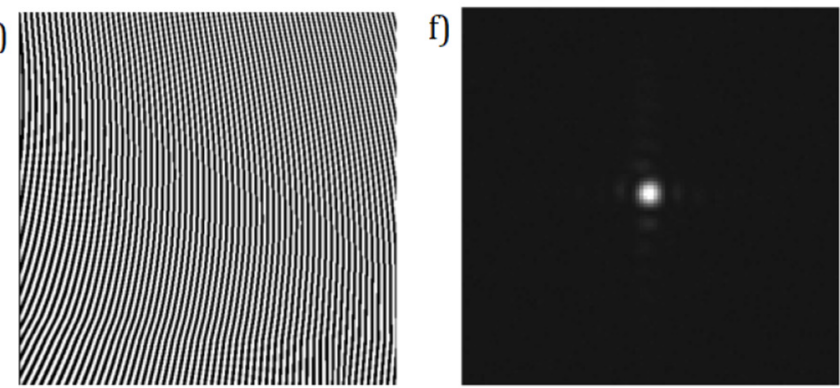

Fig. 8. Illustration of wavefront correction. Holograms projected on the DMD and corresponding patterns obtained on the Fourier plane: (a) and (b) without correction, (c) and (d) corrected after 1 iteration, (e) and (f) after 2 iterations.

8f. The profile is already satisfactory at the first iteration (Fig. 8d) but is better at the second one (Fig. 8f). An interpolation with the square of a sinc function has been performed. We then calculated the root mean square (RMS) between the results obtained and the initial sinc function. We found a deviation of about $2.3 \%$ for one iteration, and $1.1 \%$ after the second iteration. The improvement of the profile obtained after calibration of the DMD is therefore very satisfactory, even after only one iteration.

\subsection{Application to complex images}

After the above calibration of the DMD, more complex images can be displayed on the DMD, in order to record their Fourier transform on the CCD camera. We first considered Laguerre-Gaussian and Hermite-Gaussian modes (see Eq. (5)), which have the property of being their own Fourier transform [10]. The expression of these functions, determined by two positive integers $(m, n)$ are the 

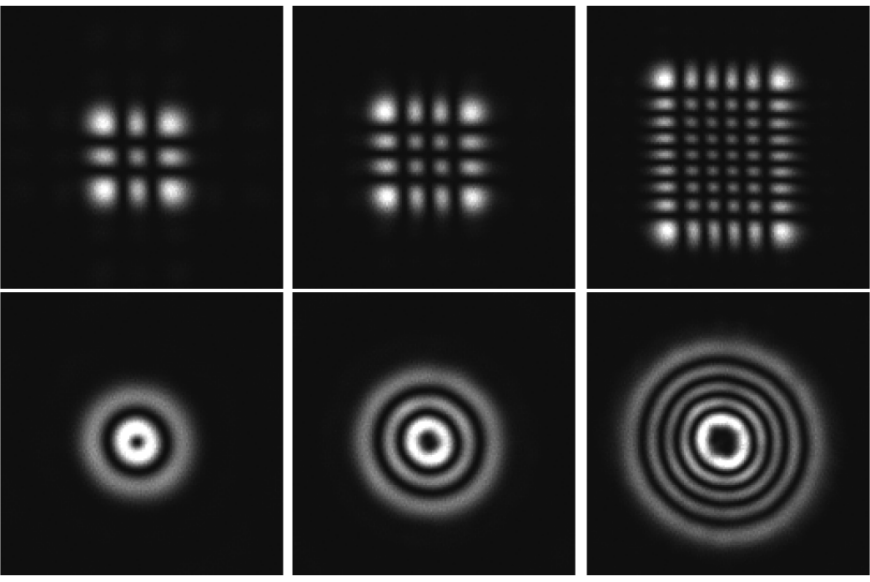

Fig. 9. Experimental restitution of $H G$ and $L G$ modes. They respectively correspond to the modes $H G_{2,2}, H G_{3,3}, H G_{5,8}$ (top) and $L G_{1,1}, L G_{2,2}, L G_{4,4}$ (bottom), from left to right.

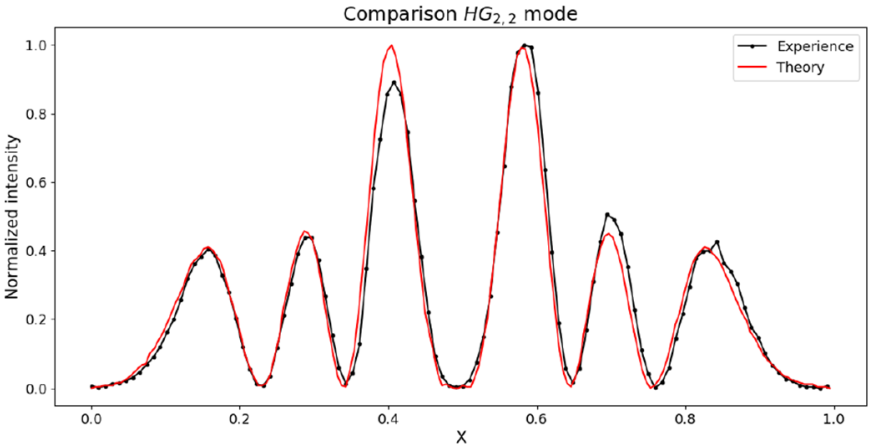

Fig. 10. Cut of the intensity profile of the mode $\mathrm{LG}_{2,2}$ in black obtained experimentally and in red as predicted by the theory. The calculated RMS error is $0.46 \%$.

following:

$$
\begin{aligned}
H G_{m, n}(x, y) & \propto H_{m}\left(\sqrt{2} \frac{x}{\sigma}\right) H_{n}\left(\frac{\sqrt{2} y}{\sigma}\right) \exp \left(-\frac{x^{2}+y^{2}}{\sigma^{2}}\right) \\
L G_{m}^{n}(r, \theta) & \propto r^{|n|} L_{m}^{n}\left(\frac{2 r^{2}}{\sigma^{2}}\right) \exp \left(-\frac{r^{2}}{\sigma^{2}}\right) e^{i \theta n},
\end{aligned}
$$

where $H_{m}$ and $L_{m}^{n}$ are respectively the Hermite and Laguerre polynomials. We used the same setup and displayed examples of corrected modes. The results are presented in Figure 9, after 1 iteration only, for the modes $H G_{2,2}, H G_{3,3}, H G_{5,8}$ (top) and $L G_{1,1}, L G_{2,2}, L G_{4,4}$ (bottom). Only order 1 , which is corrected, is depicted here. The results are already very close to the expectations. Figure 10) presents the profile of mode $H G_{2,2}$ superimposed on that of the theory, and we computed the root mean square of the normalized difference theoryexperience. The RMS error is about $0.46 \%$, which is very satisfactory.

A Fourier plane reconstitution of more complex images is also possible. As an example, we have reconstructed the image of a wind rose. The initial image is presented in Figure 11a (left), as well as its corrected Fourier transform (right). Eventually, our correction applied to the images
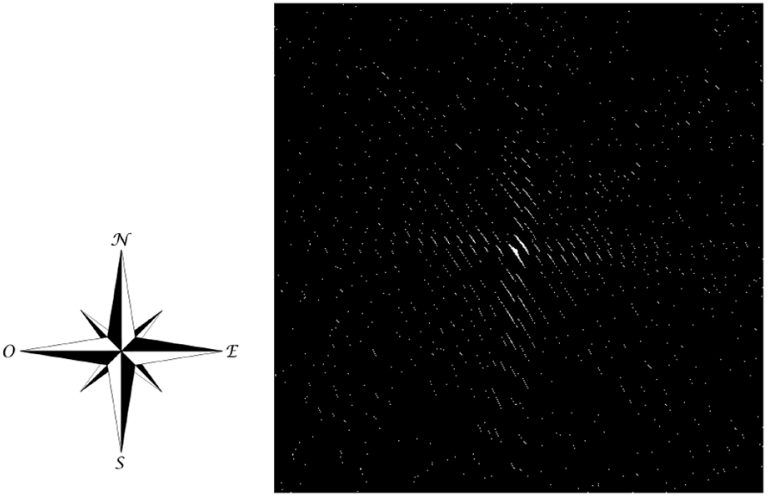

(a) initial wind rose and corrected FT
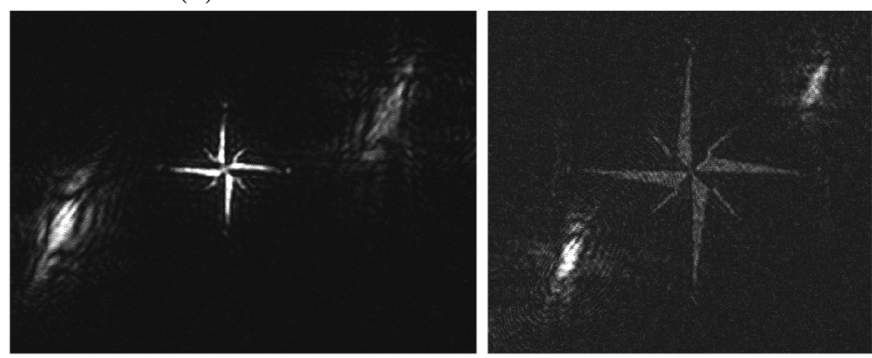

(b) Reconstitution of the Fourier plane of wind roses. The first corresponds to a rose wind smaller than the second (40 and 90 pixels for initial images, respectively).

Fig. 11. Application of the wave front correction to the wind rose image.

of the wind rose after 1 iteration leads to the results presented in Figure 11b. Here, orders 0, 1 and 2 are shown, but only order 1 is corrected and represents the wind rose image. One can notice the precision of the correction applied, since even the letters indicating the cardinal points, although small details, are visible.

We can estimate the spatial resolution of this wind rose image. By estimating several transition widths, we find $3.2 \pm 0.5$ and $2.8 \pm 0.4 \mathrm{px}$ for the small and large wind roses respectively.

\section{Discussion}

Even though these results are very satisfying, we also experienced some drawbacks and limitations with Fourier plane imaging. First, sharpness is limited by the size of the DMD, since the highest frequencies in the Fourier plane correspond to points that are further from the centre. More precisely, two aspects of the DMD must be taken into account: the spatial resolution of the image that can be obtained is inversely proportional to the total dimension of the DMD, while the total size of the image is inversely proportional to the individual size of a micromirror. Moreover, the correction scheme strongly depends on the experimental setup layout itself (including lens, CCD or DMD position) which prevents the reuse of previously established corrections and requires new calibration every time the setup is moved. Another disadvantage inherent to this method is the fact that some objects are likely to have 
a Fourier transform whose main information is located near the center of the plane (images with little details), and therefore low brightness (see Fig. 11a for the example of the compass rose). To solve this problem, it might be possible to introduce an additional random phase to the initial image. Instead of calculating the Fourier transform of an image with a phase of zero (before correction), one could calculate the Fourier transform of the image with the same amplitude, but with an additional random phase. The Fourier transform would often be more homogeneous, for an unchanged amplitude of the image. Indeed, in our gratings, the intensity is coded by the width of the holes/slots, and the phase by the interval with respect to its column or row, so that if the holes are less spaced, they are likely to be superimposed.

\section{Dead end}

Throughout these experiments, we had some difficulties with the quality of some optical components, especially the lenses: astigmatism was sometimes a problem when placing the CCD (the focusing was not done at the same distance on the horizontal and vertical plane, which is a problem to adjust the parallelism of our images when it is too important). Moreover, the indicated focal length is often wrong (sometimes up to $1 / 4$ of the focal length while the number of significant digits suggested a much smaller error). Aware of this problem, we have always measured the focal length of our lenses before using them.

Finally, with the wind rose experiment, our attempt to reconstruct larger wind roses was a failure, because the larger wind roses led us to very dark inverse Fourier transforms, which it was not possible to display on the DMD. This can be attributed to the fact that an extended object will have an inverse Fourier transform with lower frequencies since $\mathcal{F}^{-1}[f \circ(\alpha I d)](p)=\mathcal{F}^{-1}[f]\left(\frac{p}{\alpha}\right)$, so the display on the DMD is closer to the center as the homothetic factor is important, which was probably the reason why our DMD was poorly exploited. We therefore used smaller reference images to obtain more satisfactory results.

\section{Conclusion}

To summarize, our work consisted in finding the good methods and procedures to make satisfactory digital holograms in the shaping of an incident wavefront with micro-mirror devices. We succeeded in improving the spatial resolution of the recorded images by using Fourier plane imaging instead of direct imaging. Fourier plane imaging produces much sharper images, but requires a complex digital correction of the wavefront : a correction of the imperfections inherent to the surface of the device in terms of intensity and phase is necessary. The objective of the practical work (60 hours) is therefore to allow students to implement certain familiar methods and to pursue the analysis with new ideas in the domain of physical optics.

\section{References}

1. D. Gabor, A new microscopic principle. Nature 161, 777 (1948)

2. M.K. Kim, Digital Holographic microscopy (Springer, 2011)

3. P. Zupancic, Dynamic holography and beamshaping using digital micromirror devices. Master's thesis, LudwigMaximilians-Universität Munchen, 2013

4. S. Scholes, R. Kara, J. Pinnell, V. Rodríguez-Fajardo, A. Forbes, Opt. Eng. 59, 041202 (2019)

5. Y.-X. Ren, R.-D. Lu, L. Gong, Ann. Phys. 527, 447 (2015)

6. G. Gauthier, I. Lenton, N. McKay Parry, M. Baker, M.J. Davis, H. Rubinsztein-Dunlop, T.W. Neely, Optica 3, 1136 (2016)

7. A. Dareau, E. Levy, M. Bosch Aguilera, R. Bouganne, E. Akkermans, F. Gerbier, J. Beugnon, Phys. Rev. Lett. 119, $215304(2017)$

8. A. Saade, F. Caltagirone, I. Carron, L. Daudet, A. Drémeau, S. Gigan, F. Krzakala, IEEE International Conference on Acoustics, Speech and Signal Processing (ICASSP), 2016

9. M. Mansuripur, Classical Optics and its Applications (Cambridge University Press, 2002)

10. S. de Léséleuc, DMD Report, Master's thesis, Swiss Federal Institute of Technology, 2014

Cite this article as: Brice Douet, Téo Tedoldi, Adeline Kabacinski, Ambra Morana, Guilhem Gallot, Raphaël Bouganne. Digital micromirror device for holographic and Fourier optics applications, Emergent Scientist 5, 4 (2021) 